\title{
XIV.
}

\section{Ueber hyaline und amyloide Degeneration der Conjunctiva des Anges.}

Von Dr. E. Raehlmann, Professor der Ophthalmologie in Dorpat.

Sitz und Ausdehnung der Entartung.

Die Amyloiddegeneration organischer Gewebsbestandtheile ist von jeher als der Ausdruck einer Constitutionskrankheit, als ein Zeichen besonderer, für den ganzen Organismus deletärer Ernährungsprozesse aufgefasst worden. Die sämmtlichen Patbologen stimmen darin uiberein, dass die Amyloidentarlung nur in Folge besonderer dyscrasischer Blutbeschaffenheiten auftritt, und als solche für den Organismus eine unheilbare, schwere Erkrankung vorstellt.

Man fand die Amyloidbildung bisher, zwar an den verschiedensten Orten und Organen vor (Leber, Milz, Niere, den Blut- und Lymphdrüsen, den Gefässen etc. etc.) aber immer bei solchen Veränderungen des Gesammtorganismus, welche eine veränderte Blutmischung vermuthen lassen; so insbesondere nach Knochenerkrankungen, nach langwierigen Eiterungen etc. etc.

In neuerer Zeit hat nun der Nachweis von Amyloidsubstanz in der Conjunctiva des Auges besonderes Interesse hervorgerufen.

Da hier die Amyloidbildung in einem nach aussen freiliegenden Gewebstheile stattindet, fand die Forschung hier eine für die Untersuchung besonders geeigneten Boden vor, aus welchem man, ohne den Gesammtorganismus zu stören, von Zeit zu Zeit neues Beobachtungsmaterial schöpfen konnte ${ }^{1}$ ).

1) Andererseits ist das Gewehe der Conjunctiva besonders geeignet die Frage zu entscheiden, ob die Degeneration von den Zellen ausgeht, oder nicht. In den übrigen von der Amyloiddegeneration befallenen Organen wie in der Leber, Milz etc. findet sich ein sehr complicirtes 'Gewebe vollständig heterogener Gebilde neben einem grossen Gefässreichthum. In der Conjunctiva handelt es sich um ein verhältnlssmässig einfaches Gewebe von typischem Bau, es finden sich hier nur Lymphzellen in einer überall nach demselben 
Man machte hier die eigenthümliche Enfahrung, dass man Amyloiddegeneration vor sich habe, welche vollständig localisirt auftrat, obne den Organismus zu alteriren, geschweige denn zu vernichten.

Obwohl die Kliniker von den bisher bekannt gewordenen, bereits zablreichen, für die Auffassung der Amyloidentartung so böchst wichtigen Fällen keine besondere Notiz genommen haben, steht dennoch gegenwärtig das Vorkommen local begrenzter Amyloidbildung in der Conjunctiva unabhängig von Dyscrasie als eine unwiderlegliche Thatsache fest.

Es kann also unter Einfluss bestimmter Ernährungsverïnderungen, welche sicb auf ein Organ resp. einen Organtheil beschränken, aus dem normalen Eiweiss des Gewebes Amyloidsubstanz gebildet werden.

Die Amyloidbildung kann, wie die von Reymond ${ }^{1}$ ) und mir $^{2}$ ) veröffentlichten Fälle beweisen, derart circumscript auftreten, dass ein haselnussgrosser, mit der Conjunctiva zusammenhängender amyloider Tumor in seiner nächsten Umgebung nicht einmal irgend welcbe Infiltration bewirkt.

Ich habe solche Schwellungen z. B. auf die Conjunctiva bulbi beschränkt vorgefunden, während die Uebergangsfalte und die Conjunctiva der Lider vollständig gesund waren.

In dem letzten Jahre habe ich aus dem Gewebe des Oberlides einen nicht ganz bohnengrossen, scharf begrenzten Tumor exstirpirt, welcher an der Lidfläche deutlich prominirte und auch an der Conjunctivalfäche sichtbar war, somit darch die ganze Dicke des Lides sich erstreckte.

So klein dâs exstirpirte Gebilde war, zeigte es durchweg exquisite Amyloidreaction. Das Gewächs wurzelte in der Conjunctiva palpebrarum mit einer etwas über linsengrossen Basis, die äbrige Schleimbaut war vollstăndig gesund, ungetrübt, nicht einmal injicirt. Das Gewebe des Lides in der erwăbnten Grössenausdehnung war ausscbliesslich betheiligt, die umliegenden Gewebe unverändert. Das Individuam, welches den Tumor trug, war ein gesundes, kräftiges Mädchen von 19 Jahren mit durchaus gesunden Körperorganen. Dasselbe hatte sich nur der kosmetischen Verunstaltung wegen zur Eatfernung der Geschwulst entscblossen.

Bei den Patienten, welche an dem genannten Conjunctivalleiden erkrankt sind, lassen sich keine anderweitigen Störungen der Lebens-

Prinzip geformten Rüstsubstanz. Die Gefässe sind nur spärlich vorhanden. Ich glaube, dass die Frage nach dem Wesen und der Herkunft des Amyloids, wenn überbaupt, hier entschieden werden wird.

1) Reymond, Annali di Ottamologia Bd. IV. 1875. p. 349.

) Raeblmana, Archiv für Augenheilkunde Bd.X. S. 129. 
functionen, nicht einmal regelmässige Lymphdrüsenschwellungen nachweisen, es handelt sich meist um gesunde robuste Individuen aus gesunder Familie.

\section{Chemisches Verhalten des Amyloids der Conjunctiva.}

Es unterliegt somit keinem Zweifel, dass die Amyloidbildung in der Conjunctiva auf locale Krankheitsbedingungen zurückgeführt werden muss, welche unabhängig von dem Gesammtorganismus entstehen und verlaufen.

Da aber die Amyloidbildung in anderen Körperorganen als der Ausdrack bestimmter Constitutionskrankheiteo aufgefasst wird, so wird es ron grossem Interesse sein, nachzuweisen, dass die in der Conjunctiva gebildete auf $\mathrm{JSO}_{3}$ reagirende Substanz, wirklich identisch ist mit der Amyloidsubstanz der anderen Organe.

Es würde darauf ankommen, den Nachweis zu liefern, dass die erstere Substanz sich chemisch ebenso verhält, wie das von W. Kühne näher untersuchte Amyloid der übrigen Organe.

Die Analogie ist eine durchaus vollkommene.

Ich referire kurz folgende Ergebnisse der chemischen Untersuchung, welche von mir im Verein mit meinem hiesigen Collegen F. A. Hof $\mathrm{mann}$ angestellt wurden.

Die Gewebssubstanz löst sich in $\mathrm{HCl}$ in der Kälte nicht vollständig, wohl aber beim Kochen.

Beim Kochen mit $\mathrm{NO}_{5}$ entsteht ein flockig gelber Rückstand. Filtrirt und ansgewaschen zeigt er eine gallertige, fest-weiche Consisternz. Untersucht man denselben unter dem Mikroskope, so zeigt er sich bestehend aus einer glasigen, durchaus homogenen gelblichen siark lichtbrecherıden Substanz, welche mit $\mathrm{JSO}_{3}$ behandelt, sich vollständig schmutzig braunblau färbt und durch Metbyl-Violett eine intensiv rothe Farbe annimmt.

Diese Substanz stellt also das reine Amyloid vor. Dasselbe löst sich in Salzsäure beim Kochen, ebenso in $\mathbf{N H}_{3}$.

Beim Neutralisiren der salzsauren Lösung mit Natronlauge entstebt ein weisser Niederschlag.

Die ammoniakalische Lösung des reinen Amyloids coagulirt beim Kochen nicht, sie giebt mit Essigsäure ebenfalls eine Trübung, welche im Ueberschusse nicht löslich ist. 
Diese Reactionen stimmen in wesentlichen Punkten mit denen von $W$. Kühne erhaltenen überein.

Das Amyloid in der Conjunctiva ist also derselbe Körper, welcher sich auch gelegentlich in der Leber und Milz etc. vorfindet und dann von weittragender pathognostischer Bedeutung ist. Die Existenz desselben Körpers in der Conjunctiva bei sonst ganz normalem Organismus, beweist wohl zur Genüge, dass die Amyloidbildung in andern Organen, wie sie nach langwierigen Eiterungen etc. nicht selten beobachtet wird, als solche nur secundärer Natur ist, und jedenfalls das Wesen der unbekannten Prozesse, welche den Organismus bedrohen, nicht ausmacht.

Die Gewebsveränderungen bei amyloider und hyaliner Degeneration der Conjunctiva.

Das Wesen der Amyloidbildung selbst ist noch streitig. -

Das die Amyloidsubstanz durch Ausireten aus den Blutgefässen in die Gewebe abgelagert wird, wie die reine Infiltrationstheorie (Rindfleisch) es fordern würde, ist im höchsten Grade unwahrscheinlich. Man sieht nicht ein warum das Amyloid dann einzig in der Conjunctiva und gar an so umschriebenen Stellen abgelagert würde mit Ausschluss aller übrigen Körperorgane.

Der Eiweisskörper müsste vielmehr im Blute gelöst überall circuliren, auf alie Organe einwirken, was mit dem Gesundheitszustande des Individuums unvereinbar ist.

Ich stimme daher mit Cohnheim darin überein, dass das Amyloid direct aus dem präexistirenden Eiweiss entsteht, glaube aber, im Gegensatz zu Cohnheim, dass diese Degeneration von local begrenzter, von dem Zustande des Gesammtorganismus unabhängiger Gewebserkrankung, abhängig ist.

Leber (Arch. für Ophthal. Bd. XXV. I. S. 301) glaubt, dass die Amyloidbildung auf Grund einer besonderen Zellthätigkeit, speciell der Riesenzellen entstehe, und gründet auf diese Ansicht eine neue Theorie der Amyloidentartung uberhaupt.

Ich babe in dem degenerirten Gewebe keine Riesenzellen gefunden, muss auch die Genese des Amyloids als eine Art Intercellularsubstanz im Leber'schen Sinne bestreiten, indem ich die ersten hyalinen ja auch oft die ersten amyloiden Veränderungen durchweg auf die Zellen selbst beschränkt finde. Meine Auffassung 
schliesst sich vielmehr der von Böttcher und Küber u. A. gegebenen Definition an, nach welcher sich die Zellsubstanz selbst in Amyloid umwandelt.

Dem Auftreten der chemischen Amyloidsubstanz in der Conjunctiva pflegt stets ein Stadium hyaliner Degeneration vorauszugehen. Die Entstehung des Amyloids folgt bisweilen derartig rasch auf die hyaline Entartung, dass neben der Amyloidfärbung (bei Behandlung mit $\mathrm{JSO}_{3}$ ) die hyalinen Massen zurücktreten. In den meisten Fällen aber kann man neben den Amyloiddegenerirten Partien die byalinen Substanzen, welche Vorstadien der ersteren vorstellen, deutlich nachweisen.

Die ersten Anfänge der hyalinen Degeneration geben sich zu erkennen durch ein auffallendes Blasswerden der Gewebe, durch eine auffallende Transparenz derselben und durch das spätere Auftreten einer gleichmässig glasigen, bomogenen Masse. Alle diese Veränderungen sind in dem adenoiden Gewebe vorerst auf die Zellen beschränkt. Dieselben zeigen dort, wo die Degeneration im Gange ist, ein durchsichtiges Wesen, verlieren die Kerne und zeigen grosse Neigung zusa mmenzufliessen und durch ein Aneinanderlagern Klumpen zu bilden.

Wenn die hyalin gewordenen Zellen ihr granulirtes Wesen und ibre Kerne verloren baben, sind die Zellen der angrenzenden Gewebstheile im Gegensatze häufig ungewöhnlich stark granulirt. Es macht den Eindruck, wie wenn der eigentlicben Degeneration ein moleculärer Zerfall des Zellprotoplasmas vorausginge.

Die bindegewebige Rüstsubstanz widersteht anfänglich der Entartung, so dass sich die degenerirten Zellen scharf von dem Gerüste abheben, später erst geht jede Structur in einer gleichmässig degenerirten Fläche unter.

Das fibrilläre Bindegewebe der tieferen Conjunctivalsehichten insbesondere der gröberen Züge, zeigt relativ spät dieselbe Veränderung.

Die Muskelfasern der Carunkel scheinen sehr frühzeitig zu erkranken, falls der innere Lidwinkel Sitz der Degeneration ist. Auch die Gefässwandungen erkranken relativ früh, die verdickten Wandungen der kleineren Arterien stellen einen durchaus amorphen glashellen Ring vor, welcher keine Spur von Faserung erkennen lässt.

An den grösseren Arterien geht die Degeneration von der Media aus, dieselbe erscheint in der Regel schon gänzlich homogen, 
wenn die Faserung noch in den beiden anderen Gefässhäıten deutlich ausgesprochen ist, von ihr, der Med., geht die Veränderung central auf die Intima und peripher auf die Adventitia über.

Dementsprechend pflegt zu der Zeit, wenn die innere und äussere Gefässhaut hyaline, glasige Beschaffenheit zeigen und sich in $\mathrm{JSO}_{3}$ gar nicht färben, die Media bereits eine tiefbraune Farbe anzunehmen.

Die erkrankten glasigen Zellengruppen können bei ibrem Zusammenfliessen eigenthümliche wurstförmig geballte Plaques formiren, oder zu rundlichen resp. eckigen Haufen zusammenfliessen. Schliesslich geht jede Spur der Gewebszellen verloren.

Die besprochenen Veränderungen können vollständig ausgebildet sein, obne dass das Gewebe die specifische Reaction auf $\mathrm{JSO}_{\mathbf{z}}$ zeigt; doch tritt gewöhnlich auf Zusatz von Jod-Jodkaliumlösung eine hellbraune bis mahagoniartige Färbung auf, welche indess auf Zusaiz von Schwefelsäure ibren Charakter und ihre Intensilät nicht ändert.

Wenn aber die Veränderungen zu höheren Graden gediehen sind, dann werden immer inmitten des Gewebes, welches die geschilderten Veränderungen zeigt, einzelne Gewebspartien auf $\mathrm{JSO}_{3}$ intensiv blau resp. tief blau-grïn gefärbt. Durch diese Reaction documentirt sich der Uebergang der hyalinen Substanz in das eigentliche Amyloid.

Die Gewebsmassen haben bei solchem mikroskopischen Befund in der Regel eine derb speckige Consistenz. Bei ausgebreiteter Amyloiddegeneration ein hartes brüchiges Wesen.

Die Bruchfläche zeigı eine auffallende glasig-körnige Beschaflenheit.

Die ersten Anfänge der hyalinen Entartung finden sich bisweilen in vorgeschrittenen Stadien umfangreicher Tumoren, welche von der Conjunctiva ausgehen und die Structur lymphoider Geschwülste (vergl. meine Abhandlung, Archiv für Ophthalmologie Bd. X, S. 129) repräsentiren.

oft aber bildet sich die hyaline und auch die amyloide Degeneration frühzeitig in Schleimhautwucherungen von ganz geringem Umfange aus. Den Beginn der hyalinen Entartung habe ich in einfachen, von der Uebergangsfalte ausgehenden, wenig voluminösen Schleimhautduplicaturen nachweisen können. Die hyalinen Wucherungen können aber ungewöhnlich grosse Ausdehnung annehmen, bevor es zur eigentlichen Amyloidbildung kommt. 
In dem letzten von mir beobachteten Falle hatte der durch dieselben hervorgebrachte Tumor circa die Grösse eines Hühnereies. In solchen Fällen ist dann das ganze Gewebe des Lides in das Bereich der Degeneration hineingezogen (Conjunctiva, Knorpel, Muskel, subcutanes Gewebe). Das Knorpelgewebe erkrankt relativ spät, frühzeitiger die Muskelfasern. Die Letzteren zeigen bei vollständiger hyaliner Beschaffenbeit noch deutliche Querstreifung.

Auch bei vollständiger Amyloidentartung, in Fällen, wo das Gewebe auf grosse Strecken bin durch $\mathrm{JSO}_{3}$ blau gefärbt wird, sind meisteus neben den reagirenden Partien noch einzelne, oder viele, rein hyaline Stellen zu bemerken, welche die erwähnte Reaction nicht geben, höchstens sich in $\mathrm{JSO}_{3}$ leicht gelb tingiren; häufig genug sind es dann gerade die Gefässwandungen, welche innerbalb des blauen Gewebes sich mit einer glasig gelben Farbe scharf abheben.

Wenn ich nicht in vollständig hyalinen Geschwülsten bei späteren Excisionen die Amyloidbildung hätte auftreten sehen, so würde ich schon durch das blosse Nebeneinandervorkommen der genannten Veränderung auf das causale Verhältniss derselben zurückschliessen.

In einem auf meiner Klinik beobachteten Falle, welchen mein Assistent Dr. Kubly (Archiv für Ophthalmologie Bd. X. 2. S. 32) näber beschrieben bat, waren in der Conjunctiva des linken Auges eines Patienten die Zustände beginnender adenoider Wucherung vorhanden, welche ich als das Grundgewebe der fraglichen Tumoren ansehe. Am Unterlide des rechten Auges fand sich exquisite hyaline Degeneration, in der Conjunctiva des Oberlides desselben Auges exquisite amyloide Degeneration vor. An den mikroskopischen Präparaten, welche vom linken Auge stammten, sowie an denen, welche von dem Unterlide des rechten Auges herrührten, konnte an den hin und wieder sichtbaren hyalinen Partien keine Amyloidreaction nachgewiesen werden. Die Präparate aber, welche aus der Conjunctiva des Oberlides des rechten Auges stammen, färbten sich bei der Einwirkung der Jod-Jodkaliumlösung fast momentan dunkelgrünblau und ging nach $\mathrm{SO}_{3}$-Zusatz in ein mehr oder weniger reines Blau über.

Auch an den Wandungen grösserer Arterien siebt man oft alle Stadien der Entartung neben einander ausgebildet. Die Media zeigt dabei nach meinen Erfahrungen immer das am meisten vorgeschrittene Stadium, sie färbt sich an manchen Präparaten tiefblau, 
während die innere sowie die äussere Gefässhaut gelb resp. braun erscheinen. Dabei sind allmähliche Abstufungen und Uebergänge der Farben in einander zu bemerken.

Das mikrochemische Verhalten der Hyalinsubstanz.

Die Anfangsstadien des Hyalins repräsentiren homogene helle glänzende, stark lichtbrechende Massen, sie verändern auf Zusatz von Jod-Jodkaliumlösung und $\mathrm{SO}_{3}$ ihre Färbung gar nicht, sie werden höchstens schwach lichtgelb, in späteren Entwickelungsstadien aber, wie oben erwähnt, braun gefärbt.

Die Substanz, welche die hyaline Beschaffenheit zeigt, stellt offenbar einen allotropen Eiweisskörper vor, aus welchem sich das Amyloid (vielleicht durch einen Spaltungsvorgang) entwickelt.

In neuester Zeit jst von Herrn Dr. W. Uh th off ein Fall von eigenthümlicher Fettdegeneration der Conjunctiva veröffentlicht worden, in welchem offenbar fettig entartete Stellen der Conjunctiva, welche ein glasiges homogenes Wesen zeigten bei $\mathrm{JSO}_{3}$-Zusatz eine braune bis blaue Färbung annahmen. Die Präparate waren in Müller'scher Lösung conservirt.

„Die reagirenden Massen sind nach gehöriger Entwässerung der Schnitte mit absolutem Alkohol mit Chloroform extrahirbar. Ebenso nehmen dieselben beim Erwärmen einen völlig tropfbar flüssigen Zustand an. Die hier in Frage kommenden Körper stellten sich somit als eine Fettsubstanz dar."

Bei der Beschreibung dieses interessanten Falles machte der Verfasser auf die Analogie mit den bisher als Amyloid-oder Hyalindegeneration beschriebenen Fällen aufmerksam, vermuthet aber selbst, dass die Reaction der Fettsubstanz auf $\mathrm{J} \mathrm{SO}_{3}$ bedingt sei durch die Einwirkung der Müller'schen Lösung, weil dasselbe Gewebe, nachdem es eine Zeit lang in Alkohol gelegen, die Reactionsfähigkeit auf $\mathrm{JSO}_{3}$ verlor.

Wenn Jemand an die Möglichkeit einer Verwechselung der hyalinen resp. amyloiden Gewebe mit fettig degenerirten Theilen denken könnte, so sei hier ausdrücklich bemerkt, dass abgesehen von der eigenthümlichen, oben beschriebenen Reactionen die hyalinen Partien, ebenso wenig als die amyloiden beim Erwärmen flüssig werden, und sich weder in heissem Alkohol und Chloroform, noch in Aether lösen. 
Behandelt man dünne Schnitte von hyalin entarteten Präparaten unter dem Mikroskop mit concentrirter Essigsäure, so wird das ganze Präparat abnorm durchsichtig. Dort, wo die byalinen Massen liegen, tritt aber das gleichmässige hyaline Wesen deutlicher hervor, an den betreffenden Stellen sind die Zellkerne nicht zu erkennen, während sie an nicht degenerirten Stellen scharf hervortreten.

Auf gänzlich amyloid degenerirtes Gewebe hat der Zusatz von concentrirter oder verdünnter Essigsäure gar keine Einwirkung, das Gewebe wird nicht einmal durchsichtiger.

Das amyloide Gewebe, welches längere Zeit in concentrirter Essigsäure gelegen hat, zeigt noch dieselbe Reaction auf $\mathrm{J} \mathrm{SO}_{3}$.

Die hyaline Substanz quillt also in concentrirter Essigsäure leicht auf, ohne sich jedoch zu lösen. Auch in verdünnter Kalilauge verändert das Präparat sich nicht; ebenso wenig in Ammoniak.

Die hyaline Substanz ist daher auch in ihrem mikrochemischen Verhalten dem Amyloid sebr ähnlich.

Ich kann übrigens die oben citirte Beobachtung Ubthoff's, dass Fettgewebe unter Umständen eine den Amyloidstofien ähnliche Reaction zeigt, insofern bestätigen, als ich mehrfach in dem subcutanen Gewebe der Lidhaut bei amyloider Degeneration das weitmaschige Rüstgewebe, in welchem die Fettzellen eingeschlossen liegen, sammt den anliegenden Bindegewebszellen an dessen Knotenpunkten vollständig entartet fand, d. b. auf $\mathrm{J} \mathrm{SO}_{3}$ intensiv blau werden sah. An dem Inhalte der Fettzellen selbst habe ich niemals die blaue Farbe auftreten sehen.

Die Resorptionsfähigkeit der Amyloidsubstanz.

„Die grosse Resistenz des Amyloids gegen die meisten Lösungsmittel macht es begreiflich, weshalb die Amyloidsubstanz einmal in Gewebe abgelagert nicht wieder verschwindet, weshalb sie der Fäuluiss ganz widersteht, und auch bei solchen Prozessen im Organismus (Eiterungen, Brand) sich erhält, welche alle anderen Gewebstheile vernichten." (W. Kühne, Lehrbuch der physiologischen Chemie, S. 414. Leipzig 1868.)

Der vorstebende Satz charakterisirt auch noch heute den Standpunkt, welchen Pathologen und Therapeuten rücksichtlich der Heilungsfähigkeit der Amyloidentartung einnehmen.

Und in der That ist es schwer verständlich, wie ein so unlöslicher Körper wieder löslich gemacht und durch die Blut- resp. die Lymphgefässe resorbirt und aus dem Gewebe entfernt werden könnte - und doch habe ich die Rückbildung und das 
völlige Verschwinden der amyloid degenerirten Massen thatsächlich beobachten können.

In mebreren Fällen habe ich nach partiellen Excisionen vollständig degenerirter Theile, sehr ansehnliche Reste der Geschwulstmasse vollständig zurückgehen und verschwinden sehen.

In einem Falle ging die Rückbildung der Massen so rasch vor sich, dass ich den Patienten, welchen ich meinen Zuhörern mit einem wallnussgrossen Amyloidtumor behufs Excision eines kleinen Stückes vorgestellt hatte, nach einigen Wochen bereits völlig geheilt entlassen konnte. Ich habe diesen und andere Patienten nach einem halben Jahre und noch längerer Zeit wiedergesehen, and konnte mich von der Dauer der Heilung überzeugen.

Theils habe ich selbst einige Fälle (Archiv für Ophthal. Bd. X. No. 2. S. 134 u.f.) genauer beschrieben, theils hat mein früherer Assistent Dr. Kubly solche Fälle aus meiner Klinik veröffentlicht.

Der Letztere hat auch I. c. erwähnt, wie leicht in unseren Fällen das Gewebe auf die Excision reagirte.

Auch andere Autoren haben eine auffallende Verkleinerung der Geschwülste nach partieller Excision beobachtet, doch nicht in allen Fällen. Vielmehr ist von einigen Autoren ein rasches und energisches Nachwachsen sogar nach verhältnissmässig radicaler Operation berichtet worden, und mir selbst ist ein Fall dieser Art vorgekommen. Es scheint also die Möglichkeit der Rückbildung mit dem Alter der Geschwulst, resp. mit dem Grade der Degeneration zusammenzuhängen. - Wie dem auch sein möge, jedenfalls steht die Möglichkeit der Resorption der Amyloidstoffe vollständig fest.

Wenn nun kein $\mathrm{Z}$ weifel besteht, dass die Amyloidsubstanz aus den Geweben wieder schwinden kann, so liegt die Vermuthung nahe, dass dieselbe, um resorbirbar zu werden, eine bestimmte Umformung unter Einfluss gewisser Ernährungsverhältnisse erleiden muss, welche durch die entzündliche Reaction der Gewebe gegen die Excision eingeleitet werden, deren Wesen uns jedoch gegenwärtig nocb vollständig unbekannt ist.

Auf die Wichtigkeit der Thatsache aber, dass eine Resorption der Amyloidstoffe wirklich vorkommt, und auf die Consequenzen, welche diese Thatsache für die Auffassung der Amyloidentartung liberhaupt haben muss, babe ich an dieser Stelle besonders aufmerksam machen wollen. 